\title{
The Effectiveness of Group Occupational Therapy for the Cognitive Function and Independence in Children with Suicidal Ideation
}

\author{
Valda Slizyte* \\ Occupational Therapist LSMUL KK Clinic of Psychiatry, Assistant LSMUL KK Rehabilitation Clinic, Head of the Public Institution \\ Independent Children, Applied Behavioral Analysis Therapist Public Institution Behavioral Analysis, Lithuania
}

*Corresponding author: Valda Slizyte, Occupational Therapist LSMUL KK Clinic of Psychiatry, Assistant LSMUL KK Rehabilitation Clinic, Head of the Public Institution Independent Children, Applied Behavioral Analysis Therapist Public Institution Behavioral Analysis, Lithuania

\section{ARTICLE INFO}

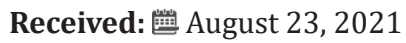

Published: 业 September 08, 2021

Citation: Valda Slizyte. The Effectiveness of Group Occupational Therapy for the Cognitive Function and Independence in Children with Suicidal Ideation. Biomed J Sci \& Tech Res 38(4)-2021. BJSTR. MS.ID.006186.

\section{ABSTRACT}

Aim: To evaluate the effectiveness of group occupational therapy for children with suicidal ideation

Tasks.

a) To establish a factor, which determines cognitive function and independence in children with suicidal ideation.

b) To evaluate the change of cognitive function while group occupational therapy is in use.

c) To evaluate the effectiveness of group occupational therapy for independence of children with suicidal ideation.

Methodology: A author-made questionnaire. The Montreal Cognitive Function Test (MoCA). The Canadian Occupational Performance Measure. For statistical analysis MS Excel was used.

Participants: 12 children with suicidal ideation tendencies were chosen for the research. The chosen target group was divided into two - research and control - groups.

\section{Conclusion:}

a) Lack of concentration, attention, motivation and quick fatigue are detrimental factors in the development of cognitive functions and independence in children with suicidal ideation.

b) Cognitive function capability improvement had statistically significant meaning $(p<0,05)$. Greater significance was noted in the study group, where group occupational therapy was implemented $(\mathrm{p}<0,05)$.

c) Independence skills had statistically significant improvement in both groups $(\mathrm{p}<0,05)$. The control group had a statistically significant improvement in general importance of functional movement, public life and active leisure $(\mathrm{p}<0,05)$ while the study group had statistically significant improvement in general satisfaction of independent activities, especially in personal and home maintenance, socialization $(\mathrm{p}<0,05)$. 


\section{Introduction}

Suicide is a deliberate interruption of your life. Unfortunately, despite a decade of trade information and prevention programs, this complex phenomenon has become the most acute problem in the modern world and the main cause of death for people of all ages is in Lithuania and the world. According to the World Health Organization (WHO), about 800,000 people die from suicide each year. Unfortunately, Lithuania has the highest number of deaths per 100,000 people in the world (31.9). Russia (31), Ukraine (22.4) and Latvia (21.2) also lead in suicide ratings. It is observed that suicides are more familiar in the former Soviet Union, middle-aged older people. According to the Lithuanian State Mental Health Center, most deaths were recorded in the 45-54 and 55-64 age groups. This suicide epidemic affects more than just suicide victims: often the suicide of a loved one has to make a psychologically negative choice for relatives, family members, friends and co-workers. Suicidal thoughts - fantasies about suicide, its idealization are especially important for the risk of suicide. It is one of the symptoms of suicidal behavior and is often directly associated with various psychological illnesses, disorders (e.g., depression, schizophrenia, alcoholism, and other addictions) [1-4]. About 10 to $20 \%$ of teens say they have persistent suicidal thoughts, which is an important indicator of predicting possible suicide attempts coming from their causes. Both suicide and suicidal thoughts are extremely important topics and problems that require public attention, and most of the information at the moment is about suicide, while suicidal thoughts are discussed in a very general way. During occupational therapy sessions, working with people at risk of suicide focuses not only on suicide attempts, but also on the thoughts that motivate or idealize it. In the absence of typical or common suicidal ideation, occupational therapists should evaluate and assign purposeful and motivating activities on a case-by-case basis. Targeted activities can be encouraged through the use of group therapy [5].

\section{Materials and Methods}

\section{Methodology}

a) A author-made questionnaire.

b) The Montreal Cognitive Function Test (MoCA).

c) The Canadian Occupational Performance Measure.

d) For statistical analysis MS Excel was used.

A. The Montreal Cognitive Function Test (MoCA) is a standardized, rapid test of cognitive function that tests memory, logical thinking, perception of time and space, abstraction, and general perception of the individual. This study is chosen for its ability to fully and quickly assess key cognitive functions. There is also a lack of studies where this test would be used in individuals under 14 years of age, then the effectiveness of this study in the same study in younger individuals. The study is divided into parts, so it is necessary to assess different cognitive abilities: attention and concentration, executive function, memory, language, visual - spatial abilities, conceptual thinking, calculation and orientation. This test is usually performed within 10 minutes. The highest evaluation is 30 points, the evaluation within the norm is desired from 26 points [6].

B. Canadian performance test is a universal form of testing that helps not only to acquire a person's independent ability, but also to set goals together with the researcher according to the most important aspects: social, personal life, work environment. The test categories are defined by functions defined by the most important aspects of life: self-care (hygiene, clothing, functional mobility, social functions: shopping, financial management, use of public transport), productivity (work, home care, science, games), leisure activities. During the survey, the respondent needs to list the most important problem activities in order to be able to perform this category and prioritize them on a scale of 1 to 10 [7].

\section{Participants}

12 children with suicidal ideation tendencies were chosen for the research. The chosen target group was divided into two research and control - groups.

The study involved 12 children aged 11 to 17 years. The following were selected according to the criteria:

a) From 11 to 17 years

b) Suicidal ideation or behavior

c) Subjects should be free of episodes of acute psychosis

d) Consent of parents, parents / guardians to participate in the study

Subjects were purposefully selected and divided into two groups: control and research. The control group, in addition to occupational therapy sessions, received regular treatment until the study group was assigned occupational therapy, during which tasks were assigned to develop independence and cognitive functions.

\section{Characteristics of the Subjects}

During the research, 12 children were studied, who were divided into two equal groups - control and research subjects. The control group received standard treatment consisting of a group of psychotherapy, psychiatric counseling, art therapy, and social skills. Occupational therapy classes and additional tasks for the researcher, focused on the development of cognitive functions and independence [8]. The mean age of the control group was 14.75 , and the mean age of the study group was -15 . In terms of symptomatology, subjects in both groups had suicidal thoughts or had committed suicidal ideation (Table 1). 
Table 1.

\begin{tabular}{|c|c|c|c|c|c|}
\hline \multicolumn{2}{|c|}{} & \multicolumn{2}{c|}{ Study group } & \multicolumn{2}{c|}{ Control group } \\
\cline { 3 - 7 } \multicolumn{2}{|c|}{} & N & Proc. & N & Proc. \\
\hline \multirow{3}{*}{ Gender } & Girls & 5 & 83,33 & 5 & 83,33 \\
\cline { 2 - 6 } & Boys & 1 & 16,67 & 1 & 16,67 \\
\hline \multicolumn{2}{|c|}{ Age } & \multicolumn{2}{|c|}{15} & \multicolumn{2}{c|}{14,75} \\
\hline
\end{tabular}

\section{Results}

After evaluating the cognitive functions by the MoCA test, it was observed that the cognitive functions of the control group increased on average by 4 points, from 20.5 to 24.5 , while the study groups evaluated the package with 5 points, from 21.5 to 26.5 . The effect of the "ceiling" of the test was also observed in the study group when one of the subjects scored the maximum allowable score (Figure 1). Different scores of scores were observed in both groups when examining scores for independence problems. Before the study, the mean score of the control group was 3.81, and that of the control group was 0.85 points lower -2.96 . This shows that before the study, the control group expressed less relevant independence problems than the study group. After the study, the mean score of the control group dropped from 0.75 points to 3.06 , while that of the study group was only 0.02 points to 2.94 . These results suggest that after the study, the representatives of the control group marked significantly more relevant independence problems, while the relevance of the research group's problems remained similar (Figure 2).

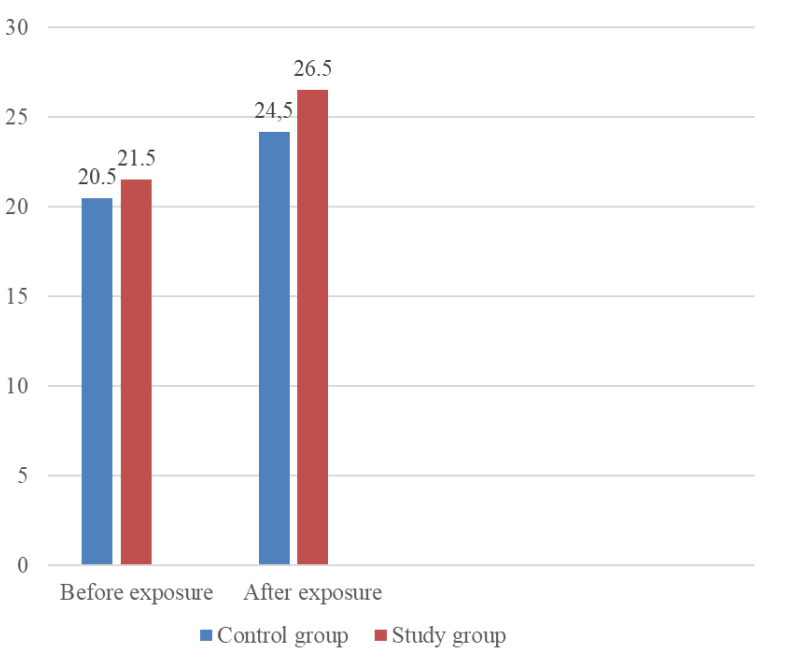

Figure 1.

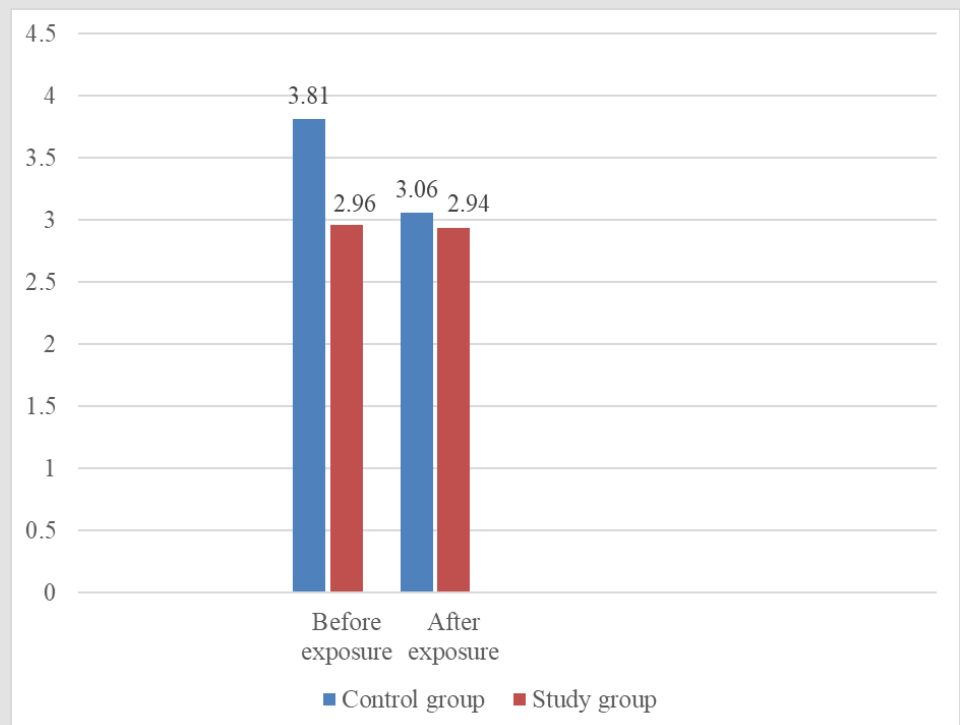

Figure 2. 


\section{Conclusion}

a) Lack of concentration, attention, motivation and quick fatigue are detrimental factors in the development of cognitive functions and independence in children with suicidal ideation.

b) Cognitive function capability improvement had statistically significant meaning $(\mathrm{p}<0,05)$. Greater significance was noted in the study group, where group occupational therapy was implemented $(\mathrm{p}<0,05)$.

c) Independence skills had statistically significant improvement in both groups $(\mathrm{p}<0,05)$. The control group had a statistically significant improvement in general importance of functional movement, public life and active leisure $(\mathrm{p}<0,05)$ while the study group had statistically significant improvement in general satisfaction of independent activities, especially in personal and home maintenance, socialization $(p<0,05)$.

\section{References}

1. Bilsen, Johan (2018) Suicide and Youth: Risk Factors. Frontiers in psychiatry 9: 540 .

\section{ISSN: 2574-1241}

DOI: 10.26717/BJSTR.2021.38.006186

Valda Slizyte. Biomed J Sci \& Tech Res

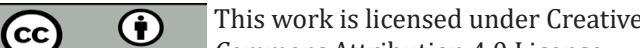
Commons Attribution 4.0 License

Submission Link: https://biomedres.us/submit-manuscript.php
2. Mousavi SG, Bateni S, Maracy MR, Mardanian F, Mousavi SH (2014) Recurrent suicide attempt and female hormones. Advanced biomedical research 3: 201.

3. Blakemore SJ, Burnett S, Dahl RE (2010) The role of puberty in the developing adolescent brain. Human Brain Mapping 31(6): 926-333.

4. Clayton PJ (2019) Suicidal Behaviour. MSD Manual, Minesotos Universiteto Medicinos Mokykla.

5. Cash Scottye J, Jeffrey A Bridge (2009) Epidemiology of youth suicide and suicidal behavior. Current opinion in pediatrics 21(5): 613-619.

6. Bilsen Johan (2018) Suicide and Youth: Risk Factors. Frontiers in psychiatry 9: 540 .

7. Dema T, Tripathy JP, Thinley S, Manju Rani, Tshering Dhendup, et al. (2019) Suicidal ideation and attempt among school going adolescents in Bhutan - a secondary analysis of a global school-based student health survey in Bhutan 2016. BMC Public Health 19: 1605.

8. Sharon D (2017) Novalis, PhD, OTR/L Assistant Professor of Occupational Therapy Chatham University Pittsburgh, PA. Suicide Awareness and Occupational Therapy for Suicide Survivors; AOTA.

\begin{tabular}{ll} 
BIOMEDICAL & Assets of Publishing with us \\
RESEARCHES & - Global archiving of articles \\
\hline Immediate, unrestricted online access \\
\end{tabular}

